\title{
Internal damping effects due to the thermal expansion mismatch between aluminium and silicon in an $\mathrm{Al} 11.8 \% \mathrm{Si}$ alloy
}

\author{
X. Zhou $\left({ }^{1,2}\right)$, R. Fougères $\left({ }^{1}\right)$ and A. Vincent $\left({ }^{1}\right)$ \\ (1) G.E.M.P.P.M., Bât. 303, Institut National des Sciences Appliquées de Lyon, 69621 \\ Villeurbanne, France \\ (2) Institute of Solid State Physics, The Chinese Academy of Sciences, China
}

(Received 13 March 1992, accepted 20 July 1992)

\begin{abstract}
Résumé. - Cette étude a été entreprise afin de développer une méthode de caractérisation du comportement des interfaces particule-matrice dans les matériaux microhétérogènes. Dans ce but, nous avons étudié au pendule de torsion le frottement intérieur (F.I.) basse fréquence dans un alliage aluminium silicium eutectique $(11,8 \% \mathrm{Si})$, présentant des particules de silicium dont la taille peut atteindre $10 \mu \mathrm{m}$. Les spectres de F.I. en fonction de la température $T$ dans le domaine $100-550 \mathrm{~K}$ sont différents au chauffage et au refroidissement de l'échantillon. Ils présentent un maximum, large et mal défini, situé autour de $240 \mathrm{~K}$ au chauffage et $190 \mathrm{~K}$ au refroidissement, ainsi qu'un fond continu croissant à haute température sur lequel est superposé un pic centré autour de $485 \mathrm{~K}$. De plus, le niveau de F.I. augmente sur l'ensemble du domaine étudié lorsque la vitesse de chauffage ou de refroidissement $|\dot{T}|$ augmente ou lorsque la fréquence de mesure est réduite. L'analyse des résultats porte essentiellement sur le phénomène dépendant de la vitesse de chauffage ou de refroidissement dont on montre que la contribution, $\delta_{T}$, provient de l'action des contraintes internes induites par la différence des coefficients de dilatation des particules de silicium et de la matrice. Nous donnons les bases d'un modèle, qui permet de décrire semiquantitativement $\delta_{\dot{T}}$. Celui-ci est fondé sur un mécanisme d'émission et de mouvement de dislocations qui serait induit par les variations des contraintes internes pendant le chauffage ou le refroidissement de l'échantillon. Le modèle prévoit une relation linéaire entre $\delta_{\dot{T}}$ et $|\dot{T}|$, qui est vérifiée de façon approchée par les résultats expérimentaux. Les autres contributions au F.I. sont discutées brièvement en terme de F.I. dû aux joints de grain, de mouvement de dislocations et de relaxation par diffusion autour des particules de silicium.
\end{abstract}

Abstract. - In order to study the behaviour of the interface between particle and matrix, low
frequency Internal Damping (I.D.) has been investigated with a torsion pendulum in an Al
11.8 wt\%Si alloy containing large silicon particles with sizes up to $10 \mu \mathrm{m}$. The I.D. spectra are
different on heating and on cooling the specimen. They exhibit a broad poorly defined maximum
situated around $240 \mathrm{~K}$ on heating and $190 \mathrm{~K}$ on cooling, and a high temperature background on
which a peak centered around $485 \mathrm{~K}$ seems to be superposed. Moreover, I.D. is increased over the
whole temperature range $100-550 \mathrm{~K}$ as the heating or cooling rate, $|\dot{T}|$, is increased, or the
oscillation frequency of the pendulum is decreased. The analysis of the experimental results is
focused on the temperature rate dependent contribution $\delta_{\dot{T}}$ that is shown to be linked with the
internal stresses induced by the difference in the coefficients of thermal expansion (CTE) of the 
silicon particles and the aluminium matrix. A model is outlined to describe $\delta_{T}$ in terms of emission or movement of dislocations that could be induced by the variation of thermal stresses during the I.D. spectrum measurement. A linear relationship between $\delta_{T}$ and the temperature rate is derived from the model and this law appears to be approximately followed by the experimental data. The other contributions to I.D. are discussed in terms of grain boundary sliding, lattice dislocation movements and diffusional stress relaxation around Si particles.

\section{Introduction.}

The interface between particle and matrix is a fundamental parameter of all microheterogeneous materials. Especially the properties of these materials derive from, and depend upon, the transfer of load from the matrix to the reinforcing second phase. Consequently, in the past, important efforts have been invested on the one hand in characterizing the interfaces and on the other hand in establishing the role of the mismatch between the coefficients of thermal expansion (CTE) of particles and matrix on the mechanical behaviour of the composite [1]. Usually, research works in this field are based not only on mechanical studies but also on optical, SEM and TEM observations. Then, despite the recent progress in modelling the macroscopic behaviour of the materials from the knowledge of the mechanical properties of the reinforcement and of the matrix [1], it remains difficult to establish a reliable relationship between the microscopic observations and the macroscopic mechanical behaviour: this difficulty arises because of the lack of experimental data dealing with the local mechanical behaviour of the interface and its surroundings, particularly when interphases or dislocations are involved in these surroundings.

As the Internal Damping (I.D.) technique is well known to be very sensitive to a large variety of irreversible movements occurring at the atomic scale, we have undertaken a study that aims at characterizing the interface and its surroundings by means of I.D. investigated as a function of temperature. This investigation is based on the fact that, because of the CTE mismatch between particles and matrix, during an I.D. experiment as a function of temperature, movements of dislocations in the neighbourhood of the interface should be induced by thermal stresses and thus should contribute to material damping.

In order to promote the potentialities of such an approach, an AI-Si alloy close to the eutectic composition has been investigated as a model microheterogeneous system.

\section{Experimental conditions.}

This study was carried out on alloys that were supplied from PECHINEY Research Center.

First, an Al-Si alloy containing $11.8 \mathrm{wt} \%$ of silicon and some minor impurities $(0.024 \mathrm{wt} \% \mathrm{Fe}, 0.005 \mathrm{wt} \% \mathrm{Ti}, 0.004 \mathrm{wt} \% \mathrm{Mg}, 0.003 \mathrm{wt} \% \mathrm{Cu}, 0.002 \mathrm{wt} \% \mathrm{Zn})$, was studied as the model microheterogeneous system. After hot-rolling, the alloy was heat-treated 40 hours at $810 \mathrm{~K}$ in order to induce a spheroidization of the Si particles. After chemical etching of the surface, SEM examinations were performed in order to examine the morphology of the Si particles. These particles appear to be quasi ellipsoidally shaped (see Fig. 1) with an aspect ratio less than 3 , and sizes up to $10 \mu \mathrm{m}$. In addition, much smaller particles were also formed during furnace cooling by precipitation of some silicon that remained in solution at $810 \mathrm{~K}$ (about $1.30 \mathrm{wt} \%$ ).

Second, some experiments were also carried out on an aluminium alloy with a lower Si content $(1.30 \mathrm{wt} \%)$. This Si content was chosen in order to obtain an alloy as similar as possible to the matrix of the eutectic alloy but without large particles formed by eutectic silicon. Such a goal was reached with an Al-Si alloy whose concentration was close to the limit of solubility of $\mathrm{Si}$ in $\mathrm{Al}$ at the temperature of furnace treatment $(810 \mathrm{~K})$. Then the concentration 


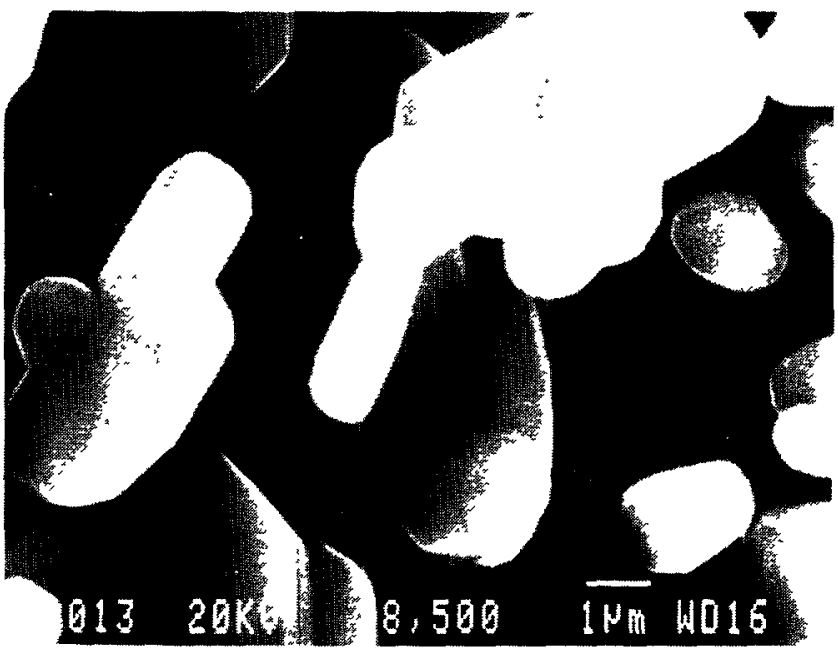

Fig. 1. - Silicon particles observed by SEM after surface chemical etching.

of silicon in solid solution in the matrix at the furnace temperature was expected to be similar in both alloys. Therefore, the matrix properties of the Al $11.8 \% \mathrm{Si}$ alloy can be obtained from the study of the Al 1.30 wt\%Si alloy.

I.D. measurements were performed with an automatic computer controlled torsion pendulum of inverted type. The oscillation frequency of this pendulum is in the range $0.6<f<4 \mathrm{~Hz}$. The system is initially forced to oscillate at an imposed amplitude, then the material damping is characterized by the logarithmic decrement $\delta$ of the freely decaying oscillations. Moreover the square of the oscillation frequency, which varies as the material modulus, will be reported each time it will be helpful to understand the material behaviour.

The measurements were carried out in the $100-550 \mathrm{~K}$ temperature range using different heating or cooling rates between $25 \mathrm{~K} / \mathrm{h}$ and $200 \mathrm{~K} / \mathrm{h}$. Unless specified, prior to each I.D. experiment the sample was maintained at the starting temperature during 2 hours.

\section{Results.}

3.1 I.D. DURING HEATING: INFLUENCE OF HEATING RATE. - The I.D. spectra of an Al 11.8\% Si sample in the annealed state are presented in figure 2 . The maximal shear strain amplitude at the surface was $5 \times 10^{-6}$ and the influence of the heating rate $\dot{T}=\mathrm{d} T / \mathrm{d} t$ has been studied in the range $25 \mathrm{~K} / \mathrm{h}-200 \mathrm{~K} / \mathrm{h}$. The typical I.D. spectrum (see curve $\mathrm{d}$ for example) exhibits a broad poorly defined maximum situated around $240 \mathrm{~K}$ and a high temperature background associated to a marked decrease in the modulus. Moreover, a high temperature peak centered around $485 \mathrm{~K}$ seems to be superposed to the high temperature I.D. background. From figure 2 it can be seen that over the whole range $100 \mathrm{~K}-550 \mathrm{~K}$, the I.D. appears to be enhanced as the heating rate $\dot{T}$ is increased. In addition, let us specify that the phenomenon is perfectly reproducible when the sample is cooled down to $100 \mathrm{~K}$ and heated again. Moreover to get a limit reference at $\dot{T}=0$, isothermal measurements were performed in steps of $10 \mathrm{~K}$ over the whole investigated range: following each temperature increment of $10 \mathrm{~K}$, the temperature of the sample was kept constant during $30 \mathrm{mn}$ in order to enable the I.D. to reach a constant value. The spectrum thus obtained is labeled « $e$ » in figure 2 . Finally, when a heating 


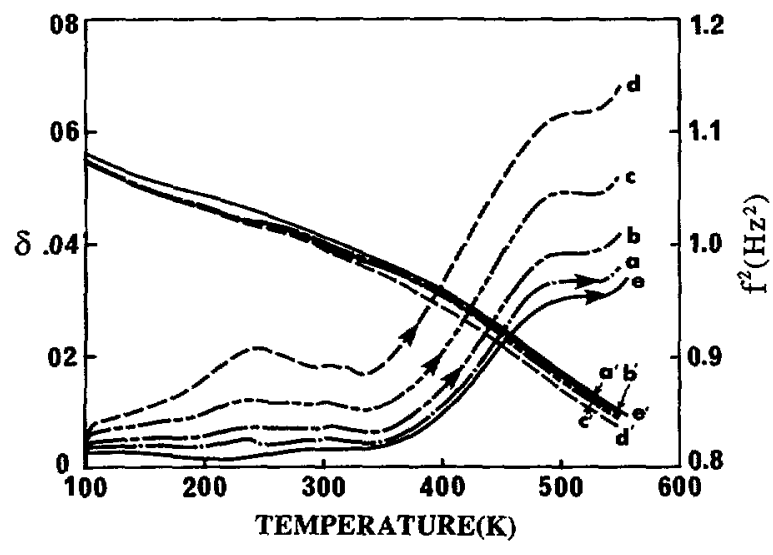

Fig. 2. - Logarithmic decrement and square of oscillation frequency versus temperature on heating for various heating rates : curves $\mathbf{a a}^{\prime}, \mathbf{b b}^{\prime}, \mathbf{c c}^{\prime}, \mathrm{dd}^{\prime}$ correspond to heating rates of $25,50,100,200 \mathrm{~K} / \mathrm{h}$, respectively. Curves ee $e^{\prime}$ were obtained by isothermal measurements. Al 11.8\%Si. Maximal shear strain amplitude $5 \times 10^{-6}$

experiment is interrupted at a given temperature, that is $T$ is kept constant at this temperature, $\delta$ decreases rapidly versus time and tends towards the level given by the spectrum « $\mathrm{e}$ » at the corresponding temperature.

In order to establish the role of the matrix in the I.D. behaviour, similar experiments were conducted on the Al 1.30\%Si alloy. The results are presented in figure $3:$ the I.D. appears to remain much smaller than that obtained in the previous case, over the whole temperature range that was investigated, especially for an high heating rate. Moreover, the I.D. is much less dependent on $\dot{T}$ than that observed in the eutectic alloy. In addition, the high temperature peak seems to be situated at a lower temperature $(450 \mathrm{~K})$ than that of the eutectic alloy.

Nevertheless, the primary conclusion derived from this comparison is that the Al $11.8 \%$ Si alloy exhibits a specific heating rate dependent I.D. that should be associated to the presence of the large silicon particles in this alloy.

Therefore, in what follows, the experimental presentation will be focused on this alloy.

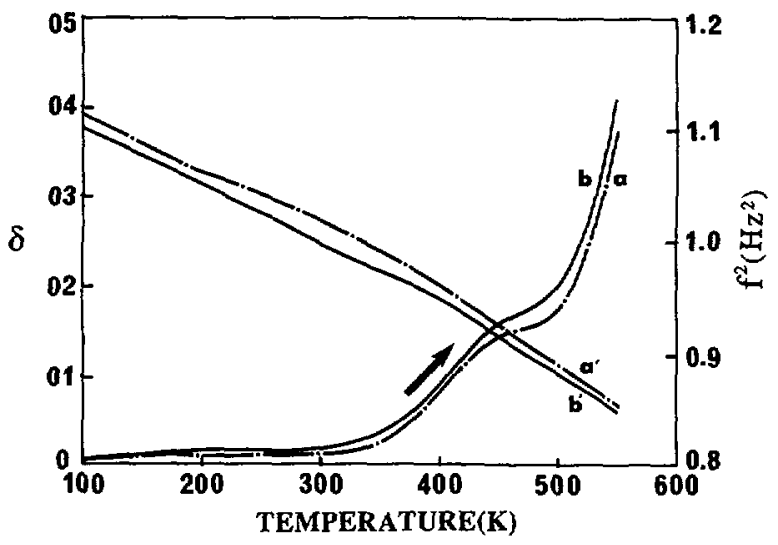

Fig. 3. - Logarithmic decrement and square of oscillation frequency versus temperature for two heating rates : curves $\mathrm{aa}^{\prime}, \mathrm{bb}^{\prime}$ correspond to 25 and $100 \mathrm{~K} / \mathrm{h}$, respectively. Al $1.3 \% \mathrm{Si}$. Maximal shear strain amplitude $5 \times 10^{-6}$ 


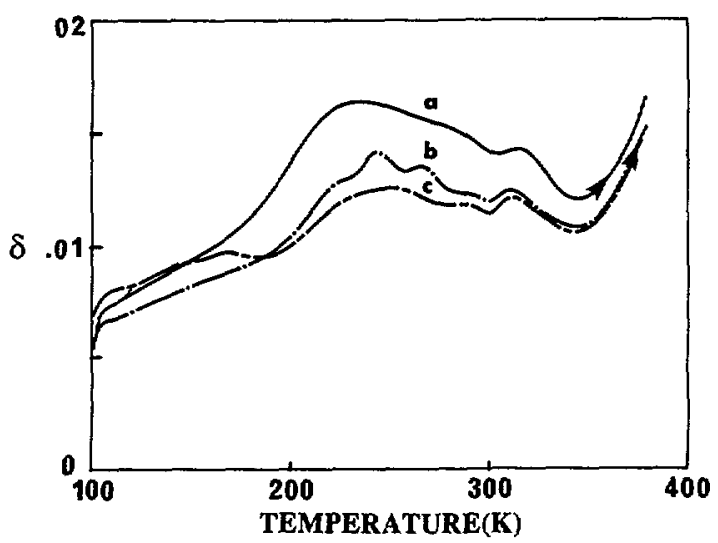

Fig. 4. - Influence of maximal strain amplitude on logarithmic decrement versus temperature : curves a, b, c correspond to shear strain amplitudes $1,5,10 \times 10^{-6}$, respectively. $\dot{T}=100 \mathrm{~K} / \mathrm{h}$. Al $11.8 \% \mathrm{Si}$.

3.2 INFLUENCE OF AMPLITUDE OF OSCILLATIONS. - The influence of the maximal shear strain amplitude at the surface of the sample has been investigated in the range $1 \times 10^{-6}-$ $1 \times 10^{-5}$. As illustrated in figure 4, in the low temperature domain and especially around the broad maximum, the I.D. is only slightly decreased when the amplitude is increased. In the high temperature domain the I.D. appears to be almost independent on the amplitude of oscillations.

3.3 INFLUENCE OF OSCILLATION FREQUENCY. - In another route, the effect of frequency of oscillations is illustrated in figure $5:$ the I.D. is enhanced when the frequency of oscillations is decreased. Moreover, similarly to what has been observed concerning the heating rate influence, the frequency influence also occurs over the whole temperature range that has been investigated.

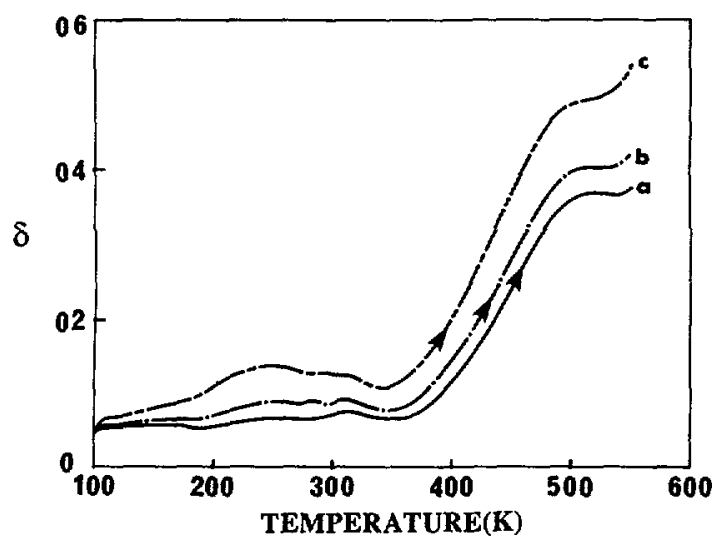

Fig. 5. - Influence of frequency of oscillation on logarithmic decrement versus temperature : curves a, $\mathrm{b}, \mathrm{c}$ correspond to frequencies (at $100 \mathrm{~K}$ ) $3.7,2.3,1.0 \mathrm{~Hz}$, respectively. Maximal shear strain amplitude $3 \times 10^{-6} \dot{T}=100 \mathrm{~K} / \mathrm{h}$. Al $11.8 \% \mathrm{Si}$. 
3.4 INTERNAL FRICTION DURING COOLING. - I.D. measurements were also performed on cooling the sample. A temperature rate effect, quite similar to that above described for heating experiments, is also observed (Fig. 6). However, at a given value of $|\dot{T}|$ the I.D. spectrum exhibits a different shape on cooling with respect to heating. For example, the spectra on heating and cooling at $|\dot{T}|=100 \mathrm{~K} / \mathrm{h}$ are displayed in figure $7:$ in the high temperature domain $(T>400 \mathrm{~K})$, the I.D. level appears to be less on cooling (curve a) than on heating (curve e), but in the low temperature domain $(T<200 \mathrm{~K})$ the contrary appears to be true.

3.5 INFLUENCE OF THERMAL CYCLING. - The influence of thermal cycling can be summarized as follows: when the sample is cycled between $550 \mathrm{~K}$ and $100 \mathrm{~K}$ the I.D. spectra are quite reproducible both on heating and on cooling.

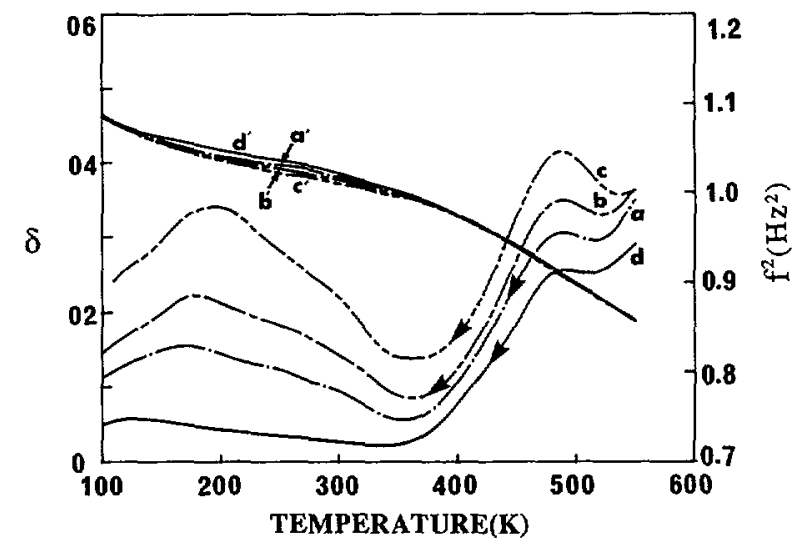

Fig. 6. - Logarithmic decrement and square of oscillation frequency versus temperature on cooling, after 24 hours at $550 \mathrm{~K}$, for various cooling rates : curves $\mathrm{aa}^{\prime}, \mathbf{b b}^{\prime}, \mathrm{cc}^{\prime}$ correspond to cooling rates 50 , $100,200 \mathrm{~K} / \mathrm{h}$, respectively. Curves dd' were obtained by isothermal measurements. Maximal shear strain amplitude $5 \times 10^{-6}$ Al $11.8 \%$ Si.

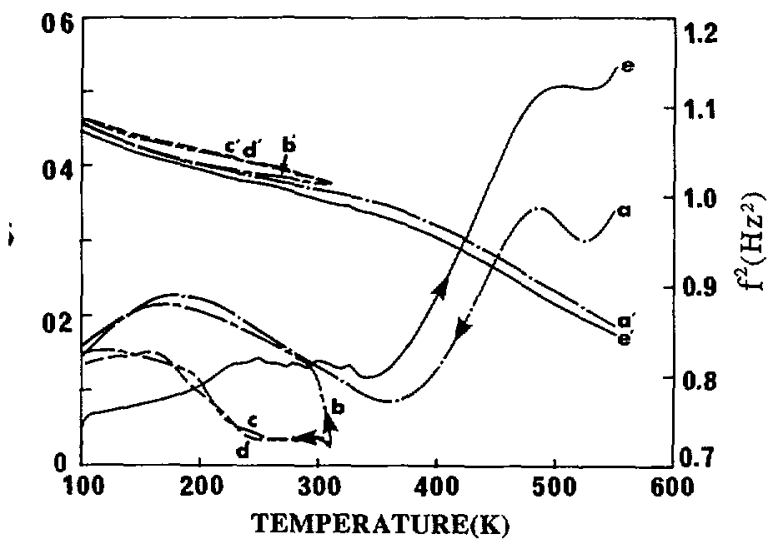

Fig. 7. - Logarithmic decrement versus temperature on cooling at $|\dot{T}|=100 \mathrm{~K} / \mathrm{h}$. Curve a, cooling from $550 \mathrm{~K}$; curve b, cooling from $320 \mathrm{~K}$; curves $\mathrm{c}$, d second, third cooling from $320 \mathrm{~K}$, respectively. Curve e recorded on heating is shown for comparison. Al $11.8 \% \mathrm{Si}$. 
However, when cycling is restricted to the low temperature domain, a marked evolution occurs between the first and the subsequent thermal cycles. This phenomenon is illustrated in figure 7 for the cooling spectra : at first, after staying 24 hours at $550 \mathrm{~K}$, the sample was cooled from this temperature, down to $320 \mathrm{~K}$ and maintained at this latter temperature during 12 hours. The subsequent I.D. spectrum recorded from $320 \mathrm{~K}$ to $100 \mathrm{~K}$ is labeled « $\mathrm{b}$ » in figure 7 : it can be seen that the I.D. increases rapidly to recover a level close to that was obtained on cooling the sample straightforwardly from $550 \mathrm{~K}$ (curve a). On the contrary, when the sample is reheated to $320 \mathrm{~K}$ and kept at this temperature for 12 hours, and then tested again on cooling down to $100 \mathrm{~K}$, the subsequent spectra (curves $\mathrm{c}$ and $\mathrm{d}$ in Fig. 7) exhibit an I.D. level that remains much lower than that observed during the first cooling (curve $b$ ).

3.6 INFLUENCE OF A PLASTIC DEFORMATION. - The sample was plastically deformed of $4.8 \%$ and $10.4 \%$ in tension at room temperature before it was set in the pendulum and cooled down to $100 \mathrm{~K}$. The influence of this deformation on the logarithmic decrement and the oscillation frequency measured on heating is reported in figure 8 . The plastic deformation seems to induce :

- a marked decrease in $\delta$ in the low temperature domain $(100-250 \mathrm{~K})$;

- the disappearance of the broad maximum previously observed around $240 \mathrm{~K}$.

On the contrary, the high temperature side of the I.D. spectrum is the more enhanced the larger the plastic deformation. Moreover in this high temperature domain, the modulus of the sample that has been normalized to its value at $100 \mathrm{~K}$ appears to decrease more rapidly in the deformed state than in the annealed one.

In addition, figure 9 shows the low temperature domain of the new spectra that were measured during a second heating of the sample subsequent to : the previous experiment, 2 hours of annealing at $550 \mathrm{~K}$, and cooling down to $100 \mathrm{~K}$. It appears that the I.D. is enhanced (curves $d$ and $e$ ) with respect to the plastically deformed state (curves $b$ and $c$ ), but it does not completely recover the level obtained in the initial state (curve a). In the high temperature domain that has not been represented, only a part of the excess of the I.D. induced by the low temperature plastic deformation appears to be recovered.

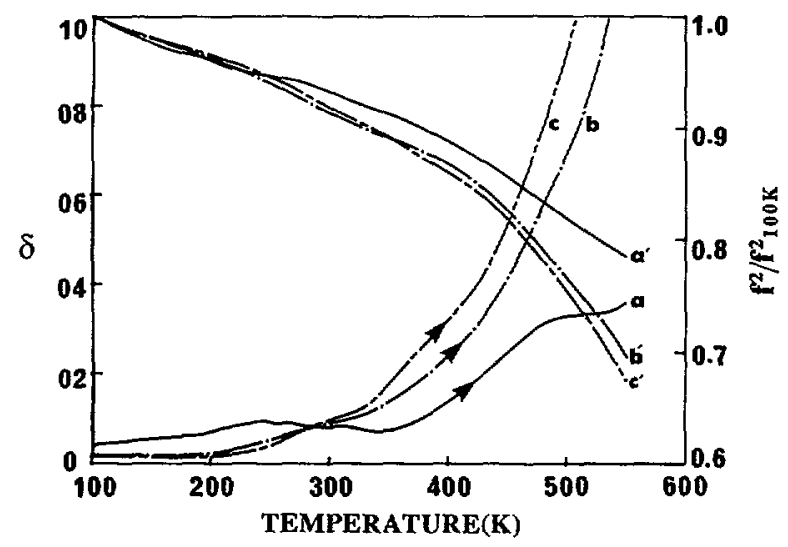

Fig. 8. - Influence of a tensile plastic deformation on the logarithmic decrement and the oscillation frequency spectra. As the measurements were carried out on different specimens, the oscillation frequency has been normalized at $100 \mathrm{~K}$; aa' annealed state ; b b' plastically deformed of $4.8 \times 10^{-2} ; \mathrm{cc}^{\prime}$ plastically deformed of $10.4 \times 10^{-2}$ Maximal shear strain amplitude $5 \times 10^{-6} \mathrm{Al} 11.8 \% \mathrm{Si}$. 


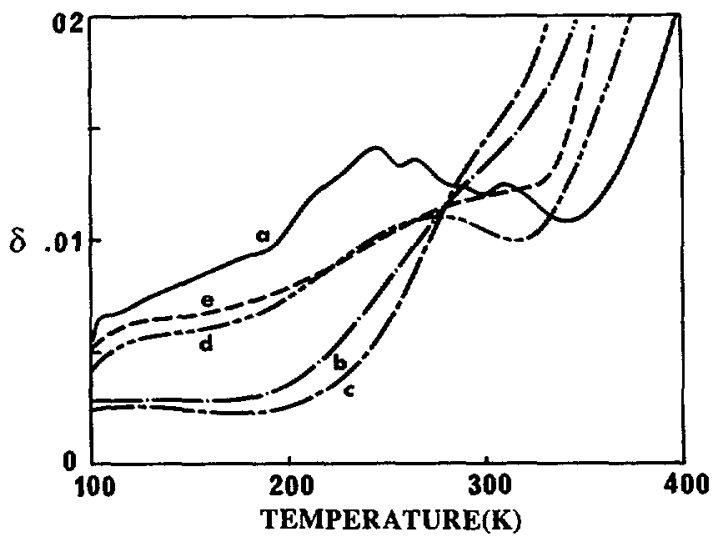

Fig. 9. - Influence of an additional annealing of 2 hours at $550 \mathrm{~K}$, subsequent to measurement after plastic deformation reported in figure 8 , on the logarithmic decrement : curves $\mathrm{d}$, e correspond to curves $\mathrm{b}$ and $\mathrm{c}$ of figure 8 . Only the low temperature domain has been reported. Spectra of the initial state (curve a) and deformed state (curves $b, c$ ) are shown for comparison. Maximal shear strain amplitude $5 \times 10^{-6}$

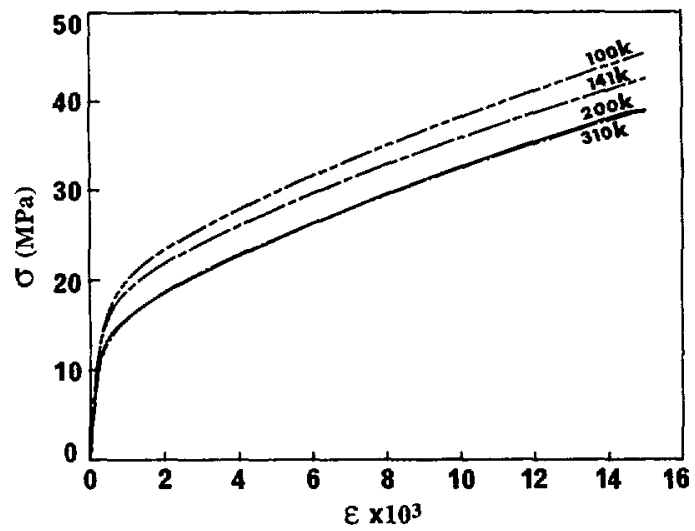

Fig. 10. - Stress-strain curves recorded during tensile tests of Al 1.3\%Si specimens, at various temperatures between $310 \mathrm{~K}$ and $100 \mathrm{~K}$.

3.7 Plastic BeHAVIOUR OF THE MATRIX. - In order to estimate the plastic behaviour of the matrix in the $\mathrm{Al} 11.8 \% \mathrm{Si}$ alloy, especially in the low temperature domain where the most striking features of I.D. have been observed, tensile tests were carried out on the Al $1.3 \% \mathrm{Si}$ alloy in the range $310 \mathrm{~K}-100 \mathrm{~K}$. The corresponding stress-strain curves are displayed in figure 10 . The yield stress appears to be independent on the temperature in the $310 \mathrm{~K}-200 \mathrm{~K}$ range, but it is significantly increased with decreasing the temperature from $200 \mathrm{~K}$ to $100 \mathrm{~K}$, which is a classical behaviour of f.c.c. metals [2].

\section{Outline of a model.}

4.1 MaIN CONTRIBUTIONS TO I.D. - In metals, I.D. can be caused by a variety of physical mechanisms including thermoelasticity, dislocation movements, stress induced point defects ordering, grain boundary sliding and so on. Moreover, in heterogeneous materials, as evoked in the introduction section, some contributions could also result from either the interface 
between particles and matrix or the particle itself. In fact, this latter contribution can be ruled out because, in the case of silicon, the mobility of dislocations is very small and, to our knowledge, in the investigated temperature range, anelastic relaxation of point defects has been observed only in doped silicon [3]. Therefore, in what follows, it will be assumed that silicon particles remain perfectly elastic and so, no energy is dissipated inside Si particles.

Moreover, since in the $\mathrm{Al} 11.8 \% \mathrm{Si}$ alloy the heating or cooling rate has been found to be a fundamental parameter, at first we shall discuss the contributions to I.D. that could explain the $\dot{T}$ effect, and then we shall consider other possible contributions to the I.D., especially to that has been measured in isothermal conditions.

Among the mechanisms that could be involved in the matrix contribution, it is expected that no influence of the heating rate should be observed for those associated with usual relaxational phenomena. To our knowledge, this parameter has been shown to be important only for non relaxational phenomena associated with phase transformation, precipitation, dissolution or recrystallization $[4,5]$. Since our experimental results have shown that the $\dot{T}$ effect occurs over a large temperature range, none of the above mentioned physical mechanisms is able to explain such a behaviour. The fact that the $\dot{T}$ influence appears to be almost suppressed in the low $\mathrm{Si}$ content alloy indicates that the interaction between the eutectic Si particles and the matrix is expected to play a major role in the $\mathrm{Al} 11.8 \% \mathrm{Si}$ alloy. Moreover, some theories have been established by various authors on the basis of an interaction between the applied stress and the particle, that is the stress concentration that results from this interaction would be relaxed by a diffusional process $[6,7]$ or by interface dislocation movements [8]. As a matter of fact these theories cannot account for the $\dot{T}$ effect because the proposed mechanisms are not expected to be influenced by $\dot{T}$. Therefore, in the next section the basis of a mechanism founded on dislocation movements mainly induced by thermal stresses due to the CTE mismatch between matrix and particles, which is effective over the whole investigated temperature range, will be proposed to account for the I.D. contribution that is dependent on $\dot{T}$.

About the isothermal I.D. background, since at low $\dot{T}$ the low temperature I.D. has been found to be very small, the discussion will be focused on the high temperature side of the I.D. spectrum. In this domain I.D. exhibits a so called high temperature background, on which a peak seems to be superposed. The following mechanisms are good candidates to contribute to I.D. in the temperature range $350-550 \mathrm{~K}$ in such aluminium alloys : grain boundary sliding, lattice dislocation movement, and the above mentioned diffusional stress relaxation process around Si particles.

At the present state of this work it is difficult to specify the respective contributions of these mechanisms to the high temperature isothermal I.D. background. Indeed, the grain boundary peak, at first observed by Kê around $550 \mathrm{~K}$ (at $f \approx 1 \mathrm{~Hz}$ ) [9] has been studied and discussed by many authors since then (see for example references [10] and [11]), in order to clarify the mechanisms at the origin of the so called Kê peak. Moreover, in the case of Al-Si alloys, it has been shown that the grain boundary peak could be shifted towards a lower temperature ( $480 \mathrm{~K}$ at $1 \mathrm{~Hz}$ ) as $\mathrm{Si}$ precipitates in grain boundaries. This phenomenon has been ascribed to an increase in the local dislocation density due to the presence of Si particles $[12,13]$. On the other hand No et al. [14] have shown that, at least in pure aluminium, the dislocation glide controlled by jog climb and vacancy pipe diffusion could explain relaxation peaks observed around $350 \mathrm{~K}$ and $440 \mathrm{~K}$. Moreover, the mechanism of diffusional stress relaxation around $\mathrm{Si}$ particles may also contribute to a relaxation peak situated in this temperature range. Indeed, Okabe et al. [6] have explained the presence of a relaxation peak situated around $420 \mathrm{~K}$ in Al 1 wt\%Si alloys by such a mechanism. Since, according to their model, the relaxation time 
associated to this mechanism, depends on the particle size, a contribution of this type of mechanism to the high temperature I.D. in our alloy cannot be ruled out.

In addition, it should be noted that other relaxation mechanisms have been observed in $\mathrm{Al}-\mathrm{Si}$ alloys but only in the quenched state that involves a high concentration of vacancies [15].

In view of the above discussion, it appears that in the Al $11.8 \mathrm{wt} \% \mathrm{Si}$ alloy, the I.D. could result from :

- a contribution, linked with the thermal stresses, that would be responsible for the $\dot{T}$ effect. This contribution will be hereafter noted $\delta_{\dot{T}}$;

- contributions, independent on $\dot{T}$, that are responsible for the background and the peak at $485 \mathrm{~K}$ observed in isothermal conditions. Their sum will be hereafter noted $\delta_{B}$.

Then, considering the fact that the contribution $\delta_{T}$ originates from the thermal stresses, which is a quite different mechanism with respect to usual I.D. mechanisms responsible for $\delta_{B}$, suggests that both ones could act in parallel, that is we could write :

$$
\delta \cong \delta_{T}+\delta_{B}
$$

4.2 BASIS OF A MODEL FOR THE HEATING RATE DEPENDENT I.D. $\left(\delta_{\dot{T}}\right)$. - Stress fields around particles that arise from the difference in CTE between particles and matrix have been studied by various authors. For instance, Brooksbank and Andrews [16] have determined the elastic stress field around spherical and cylindrical particles. This determination can also be achieved by using the Eshelby method in the case of spherical or ellipsoidal particles [1].

With a view to estimate roughly the thermal internal stresses, let us consider the case of a single spherical elastic particle in an elastic matrix : then, from [16] it can easily be shown that the maximal shear stress, $\tau_{M}$, that acts in planes oriented at $45^{\circ}$ with respect to the radial vector of the sphere, is given by:

$$
\tau_{\mathrm{M}}=\frac{3}{2} \frac{\left(\alpha_{2}-\alpha_{1}\right)\left(T_{\mathrm{a}}-T_{\mathrm{f}}\right)}{\left[\frac{\left(1+\nu_{2}\right)}{E_{2}}+\frac{2\left(1-2 \nu_{1}\right)}{E_{1}}\right]^{\frac{r^{3}}{3}}}
$$

where index 1 and index 2 refer to the particle and the matrix, respectively, and

$T_{\mathrm{a}}-T_{\mathrm{f}}=$ temperature change from the stress free state,

$\alpha \quad=$ mean coefficient of thermal expansion over the $T_{\mathrm{a}}-T_{\mathrm{f}}$ range,

$E \quad=$ Young's modulus,

$\nu \quad=$ Poisson's ratio,

$R \quad=$ radius of particle,

$r \quad=$ distance from the center of the inclusion.

In the case of the Al-Si system, by taking $[17,18]$

$$
\begin{array}{lll}
\alpha_{1}=2.6 \times 10^{-6} ; & \alpha_{2}=22.7 \times 10^{-6} ; \\
E_{1}=165 \mathrm{GPa} ; & E_{2}=70 \mathrm{GPa} ; \\
\nu_{1}=0.22 ; & \nu_{2}=0.34 ;
\end{array}
$$

it is found from relation (1) that the shear stress $\tau_{M}$ at the interface that would be induced by a temperature change, for instance of $20 \mathrm{~K}$, amounts to $23 \mathrm{MPa}$. As this value is much larger than the yield stress of the matrix (see Fig. 10) it is expected that, for I.D. measurements performed during heating or cooling, local plasticity occurs in the neighbourhood of the 
particles by means of dislocation movements as this has been shown by Hamann in the case of metal matrix composites [19]. Therefore this estimation of $\tau_{M}$ under elastic condition is a rough approximation because of the stress relaxation caused by these dislocation movements. Anyway, it is expected that the coupling between this local plastic deformation and the shear stress induced by the pendulum oscillation is responsible for $\delta_{T}$. Then, let us consider the energy density lost per cycle $\Delta w$. In a torsion pendulum the material is subjected to shear stresses that vary sinusoidally versus time. Moreover, in the case of a parallelepipedic sample, these stresses can be written as:

$$
\begin{aligned}
& \sigma_{y z}=A F_{1}(x, y) \sin \omega t \\
& \sigma_{x z}=A F_{2}(x, y) \sin \omega t
\end{aligned}
$$

where the set of coordinates is defined in figure 11 and,

$$
A=\text { amplitude of oscillations . }
$$

$F_{1}, F_{2}$ are functions that, in average, increase from the axis to the surface of the sample and exhibit local maxima near the matrix-particle interfaces.

Then the local contribution to the energy density lost per cycle of oscillation should be calculated from the expression :

$$
\Delta w=2\left[\int_{\text {cycle }} \sigma_{y z} \mathrm{~d} \varepsilon_{y z}+\int_{\text {cycle }} \sigma_{x z} \mathrm{~d} \varepsilon_{x z}\right] .
$$

From the previous considerations, that is, the local plastic deformation is ensured by movements of dislocations in the vicinity of the Al-Si interface, the local contribution, $\Delta w$, can be expressed in terms of the dislocation velocity, $v$, thus leading to :

with

$$
\Delta w=\beta b \Lambda A \int_{0}^{p} v \sin \omega t \mathrm{~d} t
$$

$P \quad=$ period of oscillation of the pendulum

$\beta(x, y)=$ geometrical and dimensional factor

$b \quad=$ length of the Burgers vector

$\Lambda \quad=$ local dislocation density

$v \quad=$ velocity of dislocation.

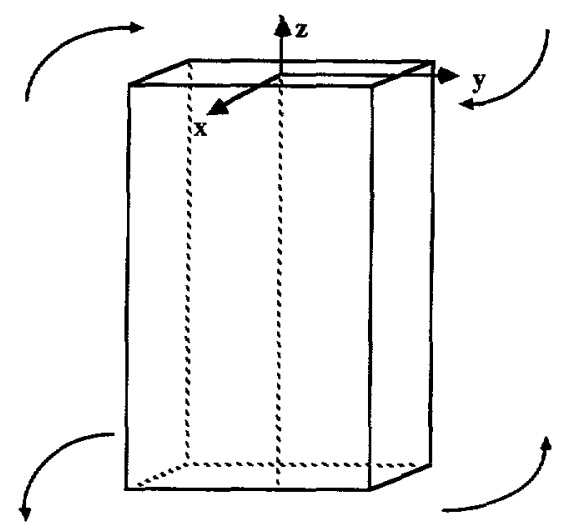

Fig. 11. - Schematic representation of the set of coordinates used to describe the externally applied stress state. 
Since the amplitude of the stress induced by the pendulum oscillation is two orders of magnitude less than the level reached by thermal stresses, the long range movements of the dislocations are expected to be mainly governed by the latter. Then, the mean velocity of dislocations, hereafter noted $v_{0}$, is controlled by the continuous evolution of the thermal stress field. However, because of the additional stress due to the pendulum oscillation, the instant velocity $v$ is expected to evolve around the mean velocity $v_{0}$. In this first approach, it is assumed that $v$ can be expressed as :

$$
v=v_{0}(1+\gamma \sin \omega t)
$$

where $v_{0}$ is the mean velocity due to the continuous evolution of thermal stresses, $\gamma$ is the factor of modulation of $v_{0}$, which is induced by the pendulum oscillation.

The detailed expressions of $v_{0}$ and $\gamma$ are difficult to establish because of the complexity of the local thermal stress field and the local effect of the applied stress. However, some fundamental trends can be derived from the following arguments. The thermally induced velocity, $v_{0}$, is expected to be an increasing function of the heating or cooling rate $|\dot{T}|$. For a sake of simplicity, a linear relationship will be considered that is :

$$
v_{0}=C|\dot{T}|
$$

where $C$ is a function of the local thermal stress and the dislocation mobility.

Note that the thermal stress is a decreasing function of the distance from the particule and it depends also on the thermal story of the sample. In addition, the dislocation mobility depends not only on the temperature, but also on the local density of dislocation.

In an another route, note that the factor of velocity modulation $\gamma$ may be smaller or greater than one according as the thermal stress is changing more or less rapidly than the applied stress, respectively. Anyway, it seems reasonable to assume that the factor of velocity modulation varies as the amplitude of oscillation $A$, that is :

$$
\gamma=d A
$$

where $d$ is a function of the coordinates $(x, y)$, the thermal stress field and the dislocation mobility.

Then, using the above expressions of $v, v_{0}$ and $\gamma$, equation (3) leads to:

$$
\Delta w=\beta C d \pi \Lambda b A^{2}|\dot{T}| / \omega
$$

Finally, the logarithmic decrement should be calculated by integrating relation (5) over the sample volume and by using the well known relation :

$$
\delta \cong \frac{\Delta W}{2 W}
$$

where $\Delta W$ is the total energy lost per cycle by the oscillating system, and $W$ is the maximal elastic energy stored during a cycle, which varies as $A^{2}$.

In view of the previous considerations, it appears that the integration over the sample volume can not be performed because the expressions of $C$ and $d$ are difficult to establish.

Nevertheless, the general form of $\delta_{T}$ can be predicted from the previous elements. Thus, in view of equations (5) and (6), $\delta_{\dot{T}}$ can be written as :

$$
\delta_{T} \propto \frac{|\dot{T}|}{\omega} F\left(\tau_{\mathrm{M}}\right. \text {, dislocation mobility, dislocation density) }
$$


where $F$ is a function that results from the integration of terms $\beta, C, d$ and $A$ of equation (5) over the sample volume.

\section{Discussion.}

Relation (7) enables us to explain the main features of the present results.

\subsection{ROLE OF EXPERIMENTAL PARAMETERS.}

5.1.1 Temperature rate dependence. - From relation (7) it appears that the contribution $\delta_{T}$ is expected to vanish at $|\dot{T}|=0$. Therefore spectra, e in figure 2 , and d in figure 6 , would represent $\delta_{B}$ in heating and cooling conditions, respectively. Then, the contribution $\delta_{\dot{T}}$ can be determined from our experimental results by subtracting $\delta_{B}$ from the total I.D. measured at various rates $|\dot{T}|$. The spectra thus obtained are shown in figures 12 and 13 in the

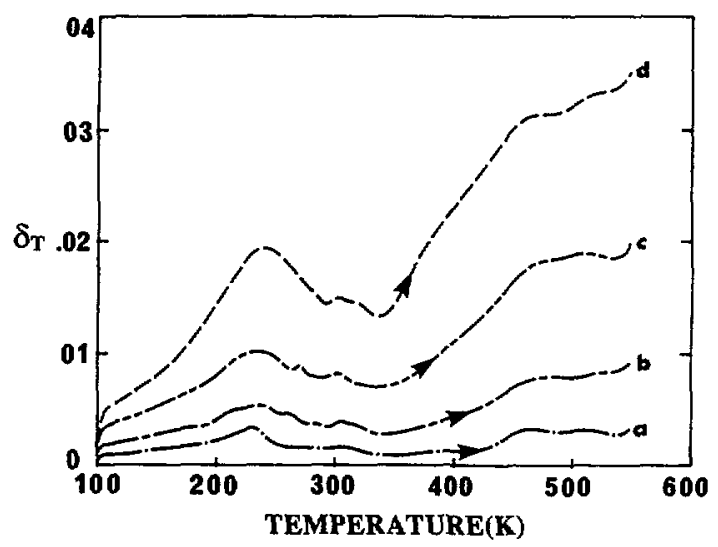

Fig. 12. - Differential spectra $\delta_{T}=f(T)$ on heating derived from figure 2 by subtracting the background contribution obtained from the isothermal measurements.

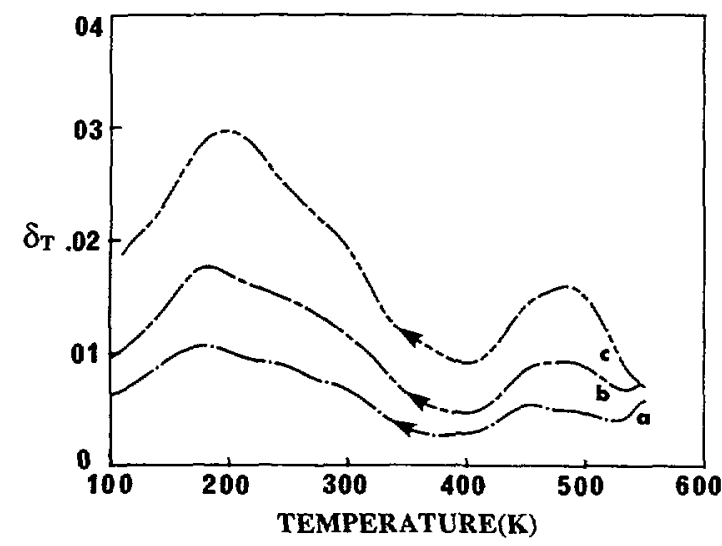

Fig. 13. - Differential spectra $\boldsymbol{\delta}_{T}=f(T)$ on cooling derived from figure 6 by subtracting the background contribution obtained from the isothermal measurements. 


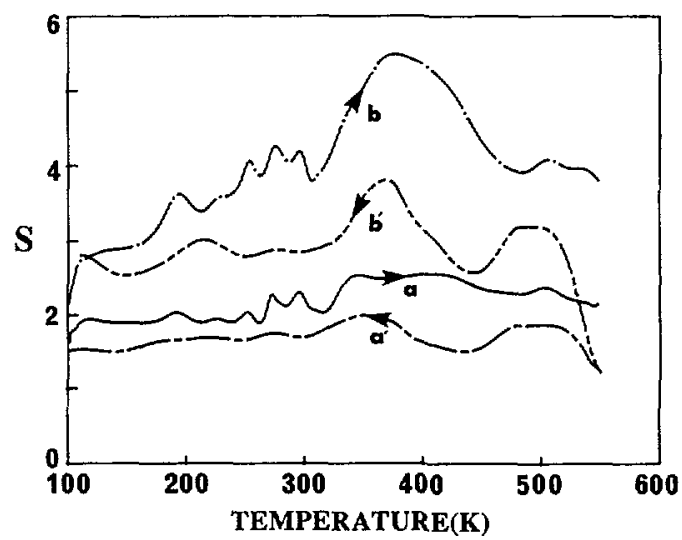

Fig. 14. - Ratio of spectra at various temperature rates : on heating

a) $\delta_{T}(\dot{T}=200 \mathrm{~K} / \mathrm{h}) / \delta_{T}(\dot{T}=100 \mathrm{~K} / \mathrm{h}) ;$ b) $\delta_{T}(\dot{T}=200 \mathrm{~K} / \mathrm{h}) / \delta_{T}(\dot{T}=50 \mathrm{~K} / \mathrm{h})$; on cooling

a') $\left.\delta_{T}(\dot{T}=200 \mathrm{~K} / \mathrm{h}) / \delta_{T}(\dot{T}=100 \mathrm{~K} / \mathrm{h}) ; \mathrm{b}^{\prime}\right) \delta_{T}(\dot{T}=200 \mathrm{~K} / \mathrm{h}) / \delta_{T}(\dot{T}=50 \mathrm{~K} / \mathrm{h})$.

case of heating and cooling experiments, respectively. Then these sets of curves $\delta_{T}=f(T)$ can be used to check the validity of the dependence of $\delta_{T}$ upon $|\dot{T}|$ predicted by relation (7). This can be done by plotting the ratio, $S$, of $\delta_{T}$ obtained for different values of the heating or cooling rate versus temperature. The result of such a treatment is presented in figure 14 for the spectrum obtained at $|\dot{T}|=200 \mathrm{~K} / \mathrm{h}$ that has been divided by the spectra obtained at $100 \mathrm{~K} / \mathrm{h}$ and $50 \mathrm{~K} / \mathrm{h}$. The experimental curves thus obtained, $S(T)$, are seen to be in reasonable agreement with relation (7). Indeed, the ratios, $S$, deduced from experimental data fluctuate between 1.5 and 2.5 (curves a and $\mathrm{a}^{\prime}$ ) when the theoretical value of $S$ predicted by relation (7) is 2 , and they fluctuate between 2.6 and 5.5 (curves $b$ and $b^{\prime}$ ) when the theoretical value is 4 .

5.1.2 Influence of frequency. - According to equation (7) $\delta_{T}$ is expected to vary in inverse ratio to the oscillation frequency. This conclusion is in qualitative agreement with the experimental results reported in figure 5.

5.1.3 Influence of amplitude. - From relation (7) it appears that the amplitude of oscillation should not influence $\delta_{T}$. This prediction is in reasonable agreement with the behaviour reported in figure 4 , that is $A$ has only a slight influence on $\delta$.

5.2 ROLE OF THERMAL INTERNAL STRESSES AND DISLOCATION MOBILITY, - The complexity of the spectra $\delta_{T}(T)$ in figures 12 and 13 arises from the evolutions of the thermal internal stresses and the dislocation mobility versus temperature, and from their combined effects. Although all this complexity cannot be explained in detail, the main features of the spectra can be understood by considering the following remarks.

5.2.1 On cooling. - During cooling, thermal internal stresses are expected to increase continuously from a very small value at $550 \mathrm{~K}$ to a maximal value at $100 \mathrm{~K}$. Indeed, the annealing at $550 \mathrm{~K}$, to which the sample is subjected prior to each cooling experiment, should enable the internal stresses resulting from the preparation to be relaxed.

In terms of mobility of dislocations two major effects have to be taken into account : first, since more and more dislocations are emitted from the $\mathrm{Si}$ particles during cooling, a forest hardening effect should occur in the vicinity of particles. Second, as shown by the tensile tests, 
the intrinsic mobility of dislocations decreases in the matrix when the temperature is lowered from $200 \mathrm{~K}$ to $100 \mathrm{~K}$. Thus, during cooling both effects tend to reduce dislocation mobility.

In consideration of these expected evolutions of the thermal internal stresses and the mobility of dislocations, the general shape of the $\delta_{T}(T)$ curves (Fig. 13) could be explained as follows. As the temperature is decreased the internal stresses are responsible, at first, for an increasing number of moving dislocations among those existing, and then, for an increasing density of dislocation, thus leading to a tendency to increase $\delta_{\dot{T}}$ versus decreasing $T$. When the effect of the dislocation mobility reduction prevails, the opposite tendency is observed. This latter situation is undoubtedly met in the range $200-100 \mathrm{~K}$, thus leading to the main maximum of I.D. observed at $190 \mathrm{~K}$. Note that the presence of the secondary maximum centered around $480 \mathrm{~K}$ is more difficult to explain.

5.2.2 On heating. - Thermal internal stresses and dislocation mobility are again expected to govern the I.D. evolutions. However, in this case they should evolve versus $T$ in a more complex manner than on cooling because of the internal stress state and the dislocation density that have been induced by cooling the sample down to $100 \mathrm{~K}$ prior to the measurements. Indeed, in the first stage of the heating experiment the internal stress level is expected to be quasi elastically reduced and no additional dislocation should be emitted from the particlematrix interface. Hence the I.D. observed in this stage would be mainly due to pre-existing dislocations whose movements are induced by this evolution of the internal stress field. Moreover, let it be mentioned that the mobility of these dislocations should be rather low.

All these remarks explain the fact that $\delta_{T}$ measured on heating (Fig. 12) remains markedly lower than $\delta_{T}$ measured on cooling (Fig. 13), in the temperature range 100-300 K.

Finally, the broad maximum observed in this range would result from the combination of the effects of, on the one hand, the complex evolution of the thermal internal stress and, on the other hand, the dislocation mobility that has been shown to increase from $100 \mathrm{~K}$ to $200 \mathrm{~K}$;

In the high temperature domain the situation is more simple because internal stresses should tend to increase again in this domain, following the above mentioned reduction. When some critical stress level is reached at the particle-matrix interface, new dislocations are emitted from this interface. Then, both the thermal expansion mismatch and the dislocation mobility evolve such that they jointly enhance dislocation emissions and dislocation displacements. These mechanisms are consistent with both the marked tendency for $\delta_{T}$ to increase in the temperature range $350-550 \mathrm{~K}$ and the high values of $\delta_{T}$ that are reached in this domain (Fig. 12) in comparison with those were observed on cooling (Fig. 13).

5.2.3 Influence of thermal cycling and plastic deformation. - The previous interpretations are well corroborated by the I.D. behaviours observed in the case of the complementary experiments dealing with thermal cycling and plastic deformation.

First, we consider the successive experiments on cooling from the intermediate temperature $320 \mathrm{~K}$.

During the annealing time at $320 \mathrm{~K}$ the internal stresses induced by the initial cooling from $550 \mathrm{~K}$ are only slightly relaxed at this temperature. Therefore, during the following first cooling from $320 \mathrm{~K}$, an internal stress level equal to that obtained on cooling straightforwardly from $550 \mathrm{~K}$ is rapidly restored, as attested by the I.D. spectrum that exhibits a steep increase of $\delta$ at the beginning of cooling (curve b in Fig. 7) followed by an evolution of $\delta$ close to that observed on cooling from $550 \mathrm{~K}$ (curve a in Fig. 7). On the contrary, for the second and the third cooling experiments, the I.D. spectrum remains markedly below that obtained during the first cooling (see curves $\mathrm{c}, \mathrm{d}$ in Fig. 7). This behaviour is undoubtedly linked with the modifications of the thermal stress field, the dislocation density, and the dislocation mobility that are induced by the first cooling, as this has been previously mentioned. 
Second, we consider the influence of the plastic deformations on the I.D. studied on heating the sample (Fig. 8).

In the low temperature domain where the contribution $\delta_{T}$ predominates, the I.D. level remains much lower in the deformed state than in the annealed state : this arises because, on the one hand, internal stresses have been at least partially relaxed by plastic deformation $[1,19]$ and, on the other hand, the matrix has been strain hardened such that dislocation movements are more difficult to initiate. As mentioned previously both effects would reduce the contribution noted $\delta_{T}$.

In the high temperature domain, matrix dislocations created by plastic deformation lead both to a marked increase in the contribution $\delta_{B}$ and to an enhanced decrease in the dynamic modulus when dislocations become more mobile.

These two remarks are well corroborated by the I.D. behaviour observed after an additional annealing at $550 \mathrm{~K}$ and a subsequent cooling to $100 \mathrm{~K}$ (see curves d, e in Fig. 9). As mentioned previously thermal stresses are expected to be relaxed during the annealing treatment at $550 \mathrm{~K}$. Then, subsequently to this additional annealing, the internal stresses would be close to that of the undeformed sample. Therefore, the $\delta_{T}$ contribution is partially restored, but the high temperature background $\delta_{B}$ would remain higher than that of an undeformed sample because of the contribution of some unannihilated dislocations.

\section{Conclusions.}

The study of Al-Si alloys as a function of temperature has shown that an important contribution to I.D. is linked with dislocation movements induced by the thermal stresses due to the CTE mismatch between the aluminium matrix and silicon particles. A semi-quantitative model has been proposed to explain the main characteristics of this mechanism of I.D. :

- $\delta_{T}$ varies as $|\dot{T}|$;

- $\delta_{T}$ is decreased when the frequency of oscillation is increased;

- $\delta_{T}$ evolutions versus temperature are governed in a complex manner by the thermal internal stress level, the dislocation density and the dislocation mobility in the vicinity of the particles.

Further studies are still required for a complete and quantitative understanding of all the observed phenomena. Nevertheless, in Al-Si alloys, I.D. measurements already appear to be a promising tool for characterizing the matrix/particle interface and its surroundings especially the dislocation behaviour linked with the thermal stress state. Moreover, this method could be beneficially applied to other microheterogeneous materials such as metal matrix composites, especially when interphases are present between matrix and particles.

\section{Acknowledgments.}

The authors gratefully acknowledge the financial support for this research provided by PECHINEY Research Center and by Region Rhône-Alpes. Thanks are also expressed to $\mathrm{Mr}$. P. Sainfort for his helpfull discussions for choosing and preparing the alloys.

\section{References}

[1] Withers P. J., Stobbs W. M. and Pedersen O. B., The application of the ESHELBY method of internal stress determination to short fiber metal matrix composites, Acta Metall. 37 (1989) 3061-3084.

[2] MCCAMmon R. D. and Rosenberg H. M., The fatigue and ultimate tensile strength of metals between 4.2 and 293 K, Proc. Roy. Soc. A242 (1957) 203-211. 
[3] BERRY B. S., Anelastic relaxation in silicon doped with lithium and boron, J. Phys. Chem. Solids 31 (1970) 1827-1834.

[4] Kiss S., Benort W., Harangozo I. Z., Kedves F. J., Posgay G. and Schaller R., Time and heating rate dependent contributions to the internal friction, J. Phys. IV France, Sup. 1248 (1987) C8-329-C8-332.

[5] KISS S., SCHAller R. and BeNOIT W., Anelastic effects due to precipitation and dissolution in AlAg alloys, Acta Metall. 34 (1986) 2151-2156.

[6] OKABE M., MORI T. and MURA T., Internal friction caused by diffusion around a second phase particle Al-Si alloy, Philos. Mag. 44 (1981) 1-12.

[7] Kohen M., Fantozzi G., Fouquet F., Merlin J., Perez J. and Gobin P. F., Relaxation phenomena due to semi-coherent precipitates, Internal friction and ultrasonic attenuation in solids, D. Lenz and K. Lucke Eds. (Springer Verlag, 1975) pp. 276-282.

[8] SchöCK G., Internal friction due to precipitation, Phys. Status Solidi 32 (1969) 651-658.

[9] KE T. S., Experimental evidence of the viscous behaviour of grain boundaries in metals, Phys. Rev. 71 (1947) 533-546.

[10] SMITH C. C. and LEAK G. M., Grain boundary damping and the high temperature background, $l l$ Nuovo Cimento 33B (1976) 388-397.

[11] Bonetti E., Evangelista E. and Gondi P., High temperature internal friction of Al, Internal Friction and Ultrasonic Attenuation in Solids, C. C. Smith Ed. (Pergamon Press, 1980) pp. 301-306.

[12] Urreta de Pereyra S. E., Bertoello H. and Ghilarducci de Salva A. A., Precipitation and grain boundary internal friction peaks in Al-Si, Phys. Status Solidi A. 108 (1988) 577-586.

[13] Urreta de Pereyra S. E., Morelli E. C. and Ghilarducci de Salva A. A., Grain boundary internal friction peak in aluminium alloys containing particles, Scripta Metall. 23 (1989) 1691 1696.

[14] No M. L., Esnouf C., SAN JUAN J. and FANTOZZI G., Internal friction at medium temperature in high purity aluminium and its relation with the microstructure (Part I and Part II), Acta Metall. 36 (1988) 827-836 (part I) 837-845 (part II).

[15] Entwistle K. M., FUller P. and Brough I., The internal friction of Al-Si alloys and the nucleation of Si precipitates, Acta Metall. 26 (1978) 1055-1059.

[16] BRooksbanK D. and ANDREWS K. W., Stress field around inclusions and their relation to mechanical properties, J. Iron Steel Institute (April, 1972) 246-255.

[17] Hahn T. A. and ARMStrong R. W., Internal stress and solid solubility effects on the thermal expansivity of Al-Si eutectic alloys, Int. J. Thermophysics 9 (1988) 179-193.

[18] NADGORNYI E., Dislocation dynamics and mechanical properties of crystals, in Progress in Materials Science, 31, J. W. Christian, P. Haasen and T. B. Massalski Eds. (Pergamon Press, 1988) p. 536.

[19] Hamann R. and Fougeres R., A model for the microplastic strains of particulate reinforced metal matrix composites, Metal Matrix Composites Processing, Microstructure and properties, N. Hansen, D. Jensen, T. Leffers, H. Lilholt, T. Lorentzen, A. S. Pedersen, O. B. Pedersen and P. Ralph Eds. (1991) pp. 373-378.

[20] HamanN R., Gobin P. F. and R. Fougeres R., A study of the microplasticity of some discontinuously reinforced metal matrix composites, Scripta Metall. 24 (1990) 1789-1794. 